Results The analysis included 3117 twin pregnancies (605 MC and 2512 DC). The total risk of early pregnancy loss (miscarriage and neonatal death) before 24 weeks in MC twins (60.3 per 1000 fetuses) was significantly higher than in DC twins (6.5 per 1000 fetuses), with a hazard ratio (HR) of 9.18 (95\% CI, 6.0-13.9). Survival analysis showed a significant difference in overall and early mortality between MC and DC twins (Log-rank test, p < 0.0001), while no difference was noted after 24 weeks of gestation (Log-rank test, $\mathrm{p}=0.08$ )

Conclusions Early pregnancy loss is significantly more common in $\mathrm{MC}$ than in $\mathrm{DC}$ twins, but the trend in prospective risk of mortality in MC twins is not evident after 24 weeks' gestation. This rate has almost halved compared to those in the published literature. Early detection and prompt treatment of complications in $\mathrm{MC}$ twins is likely to have contributed to this improvement in outcomes.

\section{PP.08 MANCHESTER ADVANCED MATERNAL AGE STUDY (MAMAS) - DOES AN AGEING MATERNAL ENVIRONMENT AND ALTERED PLACENTAL FUNCTION EXPLAIN HIGHER RISK OF POOR PREGNANCY OUT COME IN ADVANCED MATERNAL AGE?}

doi:10.1136/archdischild-2013-303966.289

SC Lean, AL Heazell, TA Mills, J Boscolo-Ryan, L Peacock, RL Jones. University of Manchester, Manchester, UK

Background Women of advanced maternal age (AMA; $\geq 35$ years) have increased risk of fetal growth restriction and stillbirth. The aetiology is unknown; however both conditions are linked with placental dysfunction, including reduced nutrient transport and altered placental morphology. Ageing is associated with increased systemic inflammation; whether this contributes to poor pregnancy outcome is unknown. We hypothesise an ageing maternal environment adversely affects placental function, resulting in poor pregnancy outcome.

Methods Women (20-30, 35-39 and $\geq 40$ years) with singleton pregnancies are being recruited to MAMAS. Maternal serum samples are collected at 28 and 36 weeks gestation for measurement of inflammatory markers by ELISA. Placental function is assessed by amino acid uptake by placental villous tissue. Placental morphology was quantified by density of Syncytial Nuclear Aggregates (SNA's), fetal capillaries and quantification of proliferation.

Results Preliminary ELISA analysis of 40 samples revealed lower anti-inflammatory cytokine interleukin-10 (IL-10) in maternal serum of women $\geq 35$ ( $p=0.016$, Kruskal-Wallis test). Other cytokines were unchanged. Preliminary data suggests higher placental uptake of taurine in women $\geq 35$, but system A activity appears unaltered. SNA's were increased, but vascularity and proliferation were unchanged in placentas from women $\geq 35(p<0.05$ Kruskal-Wallis test).

Conclusion MAMAS is the only prospective observational study investigating AMA and placental function. Preliminary data indicate accelerated placental ageing with increased SNA and an altered maternal environment with reduced anti-inflammatory cytokines. Understanding the mechanisms underlying AMA and pregnancy complications may help improve outcome for these women. Measuring circulating biomarkers of ageing prenatally may enable detection of high risk pregnancies.

\section{PP.09 FOLIC ACID SUPPLEMENTATION AND RISK OF INTRAUTERINE GROWTH RESTRICTION (IUGR)}

doi:10.1136/archdischild-2013-303966.290

${ }^{1}$ RK Morris, ${ }^{2} \mathrm{M}$ Southam, ${ }^{2} \mathrm{~J}$ Gardosi, ${ }^{1} \mathrm{~K}$ Ismail. ' University of Birmingham, Birmingham, UK; ${ }^{2}$ West Midlands Perinatal Institute, Birmingham, UK
Objective To determine whether there is a reduction in the risk of IUGR with folic acid supplementation.

Design A retrospective cohort study using the West Midlands Perinatal Institute population based database.

Setting West Midlands, UK.

Participants Births to West Midlands residents (July 2009-June 2012). Multiple pregnancies and congenital anomalies were excluded.

Main Outcome Measures Prevalence and relative risk of IUGR, defined as birth weight $<10^{\text {th }}$ customised centile with $95 \%$ confidence intervals.

Results There were $n=117260$ births with data for folic acid supplementation antenatally, of which $85 \%$ of women reported taking folic acid. Nullips constituted $42.6 \%$ of the cohort overall and $44 \%$ of those that took folic acid antenatally. For those women where the dose of folic acid was recorded $(n=42537), 95 \%$ took a dose of $400 \mathrm{mcg}, 4 \%$ at $5 \mathrm{mg}$ and $1 \%$ at other dose. For timing of folic acid supplementation, $26 \%$ commenced pre-conception, $34 \%$ at $<5$ weeks, $35 \%$ at $5-10$ weeks and $5 \%$ at a later gestation. There were $n=60077$ cases with complete pregnancy and demographic data allowing a logistic regression analysis adjusted for maternal age, smoking, hypertension, deprivation, ethnicity, employment status, diabetes (including gestational), BMI, single/partner, drug use, father blood relation, time of booking and parity. The risk of IUGR for women with no folic acid supplementation was prevalence13\%, RR 1.09 (1.03-1.16), $p<0.01$. For women that took folic acid, only the $400 \mathrm{mcg}$ dose taken pre-conception showed a significant reduction, prevalence $9.7 \%$, RR 0.90 (0.83-0.98) $\mathrm{p}=0.01$.

Conclusion Folic acid supplementation pre-conception significantly reduces the risk of IUGR.

\section{PP.10 CRL DISCORDANCE AND ADVERSE PERINATAL OUTCOME IN TWINS THE STORK MULTIPLE PREGNANCY COHORT}

doi:10.1136/archdischild-2013-303966.291

F D'Antonio, A Khalil, T Dias, A Bhide, B Thilaganathan. Fetal Medicine Unit, Division of Developmental Sciences, ST. George's Univeristy of London, London, UK

Background The role of first trimester ultrasound in predicting the outcome in twin pregnancies is conflicting. The aim of this study is to determine the association between crown-rump length (CRL) discordance and adverse perinatal outcome in twin pregnancies.

Methodology CRL discordance was related to early fetal loss $<20$, $<24$ weeks, perinatal mortality, birth weight (BW) and ultrasound estimated fetal weight (USS EFW) discordance $\geq 25 \%$, intrauterine growth restriction (IUGR) and preterm birth $<34$ weeks of gestation. ROC and logistic regression analysis was performed to evaluate the importance CRL discordance in determining adverse perinatal outcome.

Results A total of 2,155 twin pregnancies [420 monochorionic $(\mathrm{MC})$ and 1,735 dichorionic (DC)] were included in the study. CRL discordance had very poor prediction for fetal loss $<20$ (AUC of 0.61), < 24 weeks (AUC: 0.54), perinatal mortality (AUC of 0.52 ), BW discordance (AUC of 0.61 ), BW $<5^{\text {th }}$ centile (AUC of 0.56 ), USS EFW discordance (AUC of 0.55 ) and preterm birth (AUC of 0.50$)$. Overall mortality was significantly higher ( $p=0.016)$ in MC (21/420) compared to DC (45/1735) twins. Logistic regression analysis demonstrated that chorionicity ( $p=0.033$ OR: $2.09,95 \%$ C.I. from 1.06 to 4.010 ) independently contribute in determining mortality while CRL discordance $(p=0.201)$ did not. After adjusting for chorionicity, CRL discrepancy did not improve the detection of adverse outcome in either $\mathrm{MC}$ or DC twin pregnancies. 